\title{
Conjunctival Route of Administration
}

National Cancer Institute

\section{Source}

National Cancer Institute. Conjunctival Route of Administration. NCI Thesaurus. Code C38194.

Administration of a drug to the conjunctiva, the most frequently used mode of ocular drug delivery. Advantages of this topical route include direct, localized delivery to the target tissue, good accessibility into the intraocular environment owing to membrane permeability and large intercellular spaces, avoidance of hepatic first-pass metabolism, convenience, and relative painlessness. Limitations include rapid drug elimination due to lacrimation and nasolacrimal drainage, drug binding to, and metabolism by, tear proteins, and target non-specificity as a result of systemic absorption through the nasal mucosa and conjunctival vasculature. 